\title{
Outcomes of patients undergoing concurrent radical cystectomy and nephroureterectomy: A single-institution series
}

Gianpaolo P. Carpinito ${ }^{* 1}$; Grayden S. Cook ${ }^{* 2}$ Aaron N. Tverye ${ }^{2}$; Samuel A. Gold ${ }^{1}$; Yair Lotan ${ }^{1}$; Vitaly Margulis ${ }^{1}$; Jeffrey M. Howard ${ }^{1}$

${ }^{1}$ Department of Urology, University of Texas Southwestern Medical Center, Dallas, TX, United States; ${ }^{2}$ University of Texas Southwestern School of Medicine, Dallas, TX, United States

${ }^{*}$ Co-first authors

Cite as: Carpinito GP, Cook GS, Tverye AN, et al. Outcomes of patients undergoing concurrent radical cystectomy and nephroureterectomy: A single-institution series. Can Urol Assoc J 2022 February 28; Epub ahead of print. http://dx.doi.org/10.5489/cuaj.7612

Published online February 28, 2022

Corresponding author: Dr. Jeffrey M. Howard, Department of Urology, University of Texas Southwestern Medical Center, Dallas, TX, United States; jeffrey.howard@utsouthwestern.edu

$* * *$

\section{Abstract}

Introduction: Radical cystectomy (RC) and radical nephroureterectomy (RNU) are commonly performed in urological oncology. Concurrent disease in the upper tract and bladder is rare, so performing both procedures in the same setting is uncommon. Here, we report the perioperative and oncological outcomes of a single-institution series of concurrent RC+RNU.

Methods: We retrospectively reviewed the charts of patients who underwent concurrent $\mathrm{RC}+\mathrm{RNU}$ for bladder and/or upper tract urothelial carcinoma between 2006 and 2020. Patient demographic and clinical factors, perioperative parameters, and oncological outcomes were obtained.

Results: Twenty-seven patients underwent RC+RNU during the study period; 22 (81\%) were male. Median (interquartile range) patient age was 71 (67-75) years. All had a diagnosis of bladder cancer. Concurrent upper tract urothelial carcinoma (UTUC) was the indication for RNU in 12 cases (44\%) and non-functional renal unit in the remainder. Two patients (7\%) experienced early postoperative mortality. Eight patients (30\%) experienced major complications (ClavienDindo $\geq 3)$. Complications did not vary significantly between those rendered anephric $(5 / 16$, $31 \%)$ and those who were not $(3 / 11,27 \%)(\mathrm{p}=0.82$, Chi-squared test). Median (95\% confidence interval) and five-year overall survival were 47 (41-52) months and $42 \%$, respectively. Six of 22 
male patients (27\%) experienced a urethral recurrence and three of 14 patients (21\%) with nonfunctional kidneys had occult UTUC discovered on final pathology.

Conclusions: Combined RC+RNU carries an elevated perioperative risk, primarily in highly comorbid patients. Striking rates of occult UTUC in non-functional kidneys and of urethral recurrence after cystectomy were noted. $\mathrm{RC}+\mathrm{RNU}$ is an appropriate option in select patients.

\section{Introduction}

Urothelial cell carcinoma (UCC) is projected to be responsible for 84,000 new cancer cases in 2021 and to represent the $4^{\text {th }}$ most common cancer in men. ${ }^{1,2}$ A primary bladder malignancy accounts for 90-95\% of all UCC cases while upper tract urothelial carcinoma (UTUC) accounts largely for the remainder. Radical cystectomy (RC) remains the gold standard surgical treatment for invasive or pathologically adverse UCC, while radical nephroureterectomy (RNU) remains the gold standard surgical treatment for pathologically adverse UTUC., ${ }^{2,3}$

Of the approximately $25 \%$ of bladder cancer patients who have disease aggressive enough to warrant radical cystectomy, a small but appreciable fraction of patients demonstrate concurrent pathology of the upper tract that may indicate simultaneous radical nephroureterectomy. The indication for upper tract removal may be due to synchronous UTUC or non-functioning kidney(s) secondary to obstructive uropathy or intrinsic renal disease. ${ }^{2}$ Indeed, both indications are to some extent linked to the presence of UCC of the bladder cancer. Likely due to a shared pathogenesis between UTUC and UCC of the bladder, synchronous UTUC and UCC of the bladder is not uncommon. ${ }^{4,5}$ As many as $17 \%$ of those with UTUC present with synchronous bladder cancer. ${ }^{6}$

The presence of a non-functioning kidney may similarly demonstrate a relatively high prevalence in patients with aggressive UCC of the bladder. Approximately $27 \%$ of patients with muscle invasive bladder cancer present with hydronephrosis prior to cystectomy, and chronic kidney disease (CKD) patients have been shown to have significantly higher rates of multifocal UCC/UTUC. ${ }^{7-9}$ The risk of UTUC in ESRD patients is so high, approaching 50-fold higher than seen in the general population in some nations, that prophylactic bilateral nephroureterectomy of the non-functioning renal units may be considered even in the absence of UTUC when cystectomy is indicated. ${ }^{8,9}$

Still, there are relatively low proportions of patients who have severe enough pathology of both the upper and lower tracts to warrant simultaneous RC+RNU, which has hampered adequate characterization of patient outcomes. To date, only small case series and individual case reports are available on this topic. ${ }^{8,10-13}$ Accordingly, herein we present the largest case series of patients undergoing simultaneous RC+RNU in North America to date. The aim of this study is to present our single-institution experience of those cases necessitating these two procedures concurrently. Our results will help to guide counseling and management in this rare scenario. 


\section{Methods}

\section{Patient cohort}

Twenty-seven patients who underwent simultaneous radical cystectomy and unilateral or bilateral nephroureterectomy between 2006 and 2020 at our tertiary referral center institution were identified. A database including patient characteristics, operative parameters, complications, and oncologic outcomes was assembled by chart review. This retrospective review was approved by the Institutional Review Board (protocol number STU 2021-0527) and informed consent was waived.

\section{Data collection}

Patient data collected included demographic data, age at surgery, clinical comorbidities, BMI, tobacco use status, American Society of Anesthesiologists classification, history and details of prior bladder or upper urinary tract carcinoma, pre-operative cancer grade and stage, and use of neoadjuvant chemotherapy. Operative details documented included RNU laterality, surgical approach for the bladder, surgical approach for the kidney, estimated blood loss, total operative time, transfusion requirement, post-operative course details, complications using the ClavienDindo classification, and surgical pathology results. The oncologic outcomes documented included subsequent therapies, oncologic progression, urethral recurrence, last follow up, and death with associated cause.

\section{Statistical analysis}

Descriptive statistics were performed with calculation of mean with standard deviation or median with interquartile range when appropriate. Peri-operative outcomes of interest among patients rendered anephric vs. not and between different surgical approaches were calculated using the Kruskal-Wallis analysis of variance on ranks, Fisher's exact test, and chi-squared tests as appropriate. SPSS version 27 (IBM, Armonk, New York) was utilized to generate Kaplan-Meier curves for oncologic outcomes of recurrence free survival, progression free survival, cancer specific survival, and overall survival.

\section{Results}

Patient characteristics are provided in Table 1. Twenty-seven patients underwent concurrent $\mathrm{RC}+\mathrm{RNU}$ during the study period. Twenty-two patients $(81 \%)$ were male. Median (IQR) patient age was $71(67-75)$ years. All patients had a diagnosis of bladder cancer. Roughly half of patients $(14 / 27,52 \%)$ had a prior diagnosis of non-muscle invasive bladder cancer; of these, one underwent radical cystectomy due to progression to muscle-invasive disease. Concurrent UTUC was the indication for RNU in $12(44 \%)$ cases, with nonfunctional renal unit as the indication in the remaining $15(56 \%)$ cases. Eleven (41\%) patients did not require urinary diversion at the time of RC+RNU as a result of being rendered anephric. Thirteen (48\%) cases were performed via an open approach, $9(33 \%)$ by a minimally invasive approach, and the 
remaining 5 (19\%) by a combined approach (laparoscopic kidney, open bladder). Median (IQR) intraoperative transfusion of packed red blood cells was $2(0-4)$ units, estimated blood loss (EBL) was 600 (250 - 1025) mL, and operative time was 405 (379 - 441) and 346 (247 - 376) minutes for patients undergoing and not undergoing diversion, respectively. There was a significant difference in transfusions after RC+RNU by surgical approach with median (IQR) packed red blood cell transfusions of 3 (2 - 5) units for open kidney/open bladder, 0 ( 0 - 0$)$ units for minimally invasive kidney/open bladder, and $1(0-2)$ units for minimally invasive kidney/minimally invasive bladder approaches. Otherwise, no significant differences were found in peri-operative outcomes by surgical approach (Table 3). Eleven (41\%) patients were rendered anephric by the procedure. Median postoperative length of stay was 6 (4.5 - 9) days. Two (7\%) patients experienced early postoperative mortality, one due to a myocardial infarction and the other due to rapidly progressive sepsis from a deep surgical site infection. Notably, these deaths were in the two patients with the highest preoperative Charlson-Deyo comorbidity scores ( 9 and 10, respectively). Eight (30\%) patients experienced a Clavien-Dindo grade 3 or 4 complication. The rate of major (Clavien-Dindo grade $\geq 3$ ) complications did not vary significantly between those rendered anephric by the procedure $(5 / 16,31 \%)$ and those who were not $(3 / 11,27 \%)(p$ $=0.82$, chi-square test).

While only one patient (3.7\%) had preoperative clinical node positive status, seven (26\%) patients had node positive disease on final pathology, two of whom had received neoadjuvant chemotherapy. Of the fourteen patients who had RNU performed for nonfunctional kidneys, three (21\%) were found to have occult UTUC on final pathology (one pTa, two pT3). Three (11\%) patients had a positive surgical margin: one with carcinoma in situ at the urethral margin, one with carcinoma in situ at the left distal ureteral margin, and one with pT4b disease and a positive perivesical soft tissue margin. Fourteen patients (52\%) were alive at last follow-up, with median follow up of $36(11-52)$ months. Median $(95 \% \mathrm{CI})$ and 5-year overall survival were 47 (41 - 52) months and $42 \%$, respectively. Median $(95 \% \mathrm{CI})$ and 5-year progression-free survival were $30(16-44)$ months and 41\%, respectively. Notably, 6 of 22 male patients (27\%) experienced a urethral recurrence requiring subsequent urethrectomy, of whom only one had a positive urethral margin at the time of cystectomy. Of these, four had RNU performed for UTUC; a fifth had RNU performed for a nonfunctional kidney and was unexpectedly found to have UTUC on final pathology.

\section{Discussion}

Urothelial cancer is the fourth most common cancer in men. While UTUC accounts for only 5$10 \%$ of all urothelial cancer, it does frequently co-occur with UCC of the bladder. Radical nephroureterectomy and cystectomy, respectively, constitute the gold-standard therapies for the most severe local forms of each individual malignancy. Reports of simultaneous RC+RNU are limited, however, due to the rarity of clinical scenarios necessitating the procedure. 
In this study, we present the largest US case-series of patients undergoing RC+RNU. Mortality within 90 days of surgery was $7 \%$ and occurred in the two patients with the highest CCI. Meanwhile, grade III-IV complications were $30 \%$ and did not vary between those rendered anephric by the procedure and those who were not. A notable proportion of patients with nonfunctional kidneys $(3 / 14,21 \%)$ had occult UTUC discovered on final pathology. Most interestingly, we observed a particularly high rate of urethral recurrence (27\%) among male patients. Taken together, our results suggest that with careful patient selection, concurrent $\mathrm{RC}+\mathrm{RNU}$ may be safely performed in most patients. Likely due to underlying disease-related factors, urethral recurrence appears to be high, and providers may emphasize potential need for subsequent urethrectomy in counseling patients and close surveillance of the urethra. Our findings also emphasize the importance of vigilance for occult UTUC in patients with UCC of the bladder and concurrent hydronephrosis.

Peri-operative outcomes did not differ greatly from those reported in the isolated RC or combined RC+RNU literature. Notably, the two patients experiencing perioperative death possessed the highest CCIs of all patients in the study ( 9 and 10, respectively). Interestingly, our mortality rate was not dissimilar from the reported range of 4-6\% for patients $>75$ years of age or with severe comorbidities undergoing radical cystectomy. ${ }^{14-16}$ Previous studies on combined $\mathrm{RC}+\mathrm{RNU}$ are heterogeneous, such that reported mortality rates range from $0-25 \% .{ }^{8,10-13}$ The observed 90-day major complication rate (Clavien-Dindo $\geq 3$ ) of 30\% falls near the high end of the range of $15-27 \%$ outlined in radical cystectomy series. ${ }^{3,15,17,18}$ Similarly, our 90-day readmission rate of $26 \%$ is similar to that reported in the RC literature. ${ }^{19}$ Nephroureterectomy is a far less morbid procedure with peri-operative mortality rates of $<2 \%$ and major complications of $7-15 \%$, suggesting that radical cystectomy disproportionately drove adverse outcomes in this study ${ }^{20}$ It is important to note that the cited outcomes data were drawn from a wide variety of time periods and settings, and we are unable to provide a direct comparison with the outcomes of patients undergoing RC without RNU at our institution during the same time period. Thus, while our morbidity outcomes appear generally similar to those reported for patients undergoing $\mathrm{RC}$ alone, our comparisons of the morbidity of $\mathrm{RC}$ and $\mathrm{RC}+\mathrm{RNU}$ are necessarily speculative.

Median and 5-year overall survival in this study were 47 months and $42 \%$, respectively, though direct comparisons with prior studies are difficult due to the unique population studied here. That said, median overall survival following nephroureterectomy for UTUC in a large study was reported at 24 months. ${ }^{21}$ Median overall survival for radical cystectomy has been reported at as low as 22 months and as high as 70 months with a 5-year overall survival rate of about $50 \% .^{16,18}$ Previous series of RC+RNU reveal similarly disparate long-term survival outcomes. ${ }^{8,10-13}$ Our study population had a comparatively large proportion of patients with nonmuscle invasive clinical staging within the bladder (cTa/Tis/T1), but a large proportion of these had pan-urothelial disease affecting the upper tract as well. Taken together, RC+RNU likely can be approached when indicated without severely endangering long-term survival outcomes. 
We further noted that patients rendered anephric by the procedure did not demonstrate higher perioperative morbidity than those who did not, suggesting that in the immediate postoperative period, the increased risk of complications associated with the anephric state may be partially balanced by reducing the risk of morbidity associated with urinary diversion. Interestingly, a previous study of ESRD patients found significantly improved overall survival in patients who underwent bilateral nephroureterectomy at the time of cystectomy, though the underlying reason was unclear. ${ }^{11}$ We noted that a particularly high proportion of patients $(21 \%)$ undergoing simultaneous RNU for non-functional kidneys possessed occult UTUC. It is possible, especially in ESRD patients, that RNU may have a protective effect due to elimination of present and future risk of progressive UTUC. In light of these findings and the limited benefit of retaining a non-functional kidney, one should consider RC+RNU for patients with bladder cancer and non-functional kidney(s).

Perhaps most interestingly, a high urethral recurrence rate of $27 \%$ was observed among male patients in this study, while previous reviews of radical cystectomy patients summarize the rate of urethral recurrence at 4-6\%. ${ }^{22-24}$ A majority of our urethral recurrence patients had a history of smoking or UTUC and all had non-muscle invasive disease, all of which are reported risk factors for urethral recurrence. ${ }^{22,23,25,26}$ A potential explanation for the high urethral recurrence rate is an increased risk of recurrent malignancy due to CKD, which has been shown to increase the risk of recurrence in the bladder following nephroureterectomy. ${ }^{9} \mathrm{CKD}$ was notably present in all patients with urethral recurrence and had an overall high prevalence in our cohort. Likewise, the co-occurrence of UTUC with UCC of the bladder may imply a more severe field effect of the entire urothelium, including the urethra. This is potentially supported by the fact that three of patients with urethral recurrence and prior UTUC had previously undergone contralateral RNU prior to combined RC+RNU. These patients therefore all eventually developed metachronous pan-urothelial disease. Notably, only one out of six patients with urethral recurrence had a positive urethral margin at the time of cystectomy, suggesting that margin-positive status is not the primary determinant of urethral recurrence. Ultimately, though the underlying reasons for this finding appears unclear, we would suggest emphasizing the potential for urethral recurrence in these patients and considering performing concurrent urethrectomy at the time of surgery.

\section{Limitations}

There are several limitations to our study. Due to this study's observational, retrospective design, the strength of the conclusions that can be drawn from these results is limited. The study period spanned a long time period, encompassing numerous changes in management practices and available treatment options. Accepting this, the goal of this study was to identify basic information to assist with clinical decision-making and counseling. Because of the uncommon coincidence of indications for simultaneous RC+RNU, our sample group was small and may predispose our findings to the effect of chance. This is an inherent problem in research of this 
topic but could be addressed in the future by pooling of data from multiple institutions. Finally, it is important to note that all surgeries were conducted at a single high volume academic center, thus limiting the generalizability of our findings to other settings.

\section{Conclusions}

We present one of the largest published series of outcomes following concurrent RC+RNU. Our observed complication rates are comparable to those previously described for radical cystectomy alone, though we are unable to provide a direct comparison of the morbidity of RC+RNU and $\mathrm{RC}$ alone. Further, a substantial proportion of patients can achieve long-term recurrence-free and overall survival following concurrent $\mathrm{RC}+\mathrm{RNU}$, making it an appropriate option in management of carefully selected patients. Patients with non-functional kidneys at the time of cystectomy for bladder cancer may have a higher rate of occult UTUC. A strikingly elevated rate of urethral recurrence was noted among male patients undergoing $\mathrm{RC}+\mathrm{RNU}$, suggesting a unique panurothelial disease state in these patients and emphasizing the importance of urethral surveillance. 


\section{References}

1. Siegel RL, Miller KD, Fuchs HE, et al. Cancer statistics, 2021. CA Cancer J Clin 2021;71:7-33.

2. Rouprêt M, Babjuk M, Burger M, et al. European Association of Urology Guidelines on upper urinary tract urothelial carcinoma: 2020 update. Eur Urol 2021;79:62-79.

3. Chang SS, Bochner BH, Chou R, et al. Treatment of non-metastatic muscle-invasive bladder cancer: AUA/ASCO/ASTRO/SUO Guideline. J Urol 2017;198:552-559.

4. Hafner C, Knuechel R, Zanardo L, et al. Evidence for oligoclonality and tumor spread by intraluminal seeding in multifocal urothelial carcinomas of the upper and lower urinary tract. Oncogene 2001;20:4910-4915.

5. Miyake H, Hara I, Kamidono S, et al. Multifocal transitional cell carcinoma of the bladder and upper urinary tract: Molecular screening of clonal origin by characterizing CD44 alternative splicing patterns. J Urol 2004;172:1127-1129.

6. Cosentino M, Palou J, Gaya JM, et al. Upper urinary tract urothelial cell carcinoma: Location as a predictive factor for concomitant bladder carcinoma. World J Urol 2013;31(1):141-145.

7. Zhu Z, Zhao J, Li Y, et al. Prognostic value of preoperative hydronephrosis in patients with bladder cancer undergoing radical cystectomy: A meta-analysis. PLoS ONE [serial on the Internet]. 2019 [cited 2021 Sept 13];14(9):[12p.].

https://doi.org/10.1371/journal.pone.0222223. Accessed September 13, 2021

8. Ou Y-C, Yang C-R, Yang C-K, et al. Simultaneous robot-assisted nephroureterectomy and cystectomy in patients with uremia and multifocal urothelial carcinoma. $J$ Endourol 2011;25:979-984.

9. Chung SD, Huang KH, Lai MK, et al. CKD as a risk factor for bladder recurrence after nephroureterectomy for upper urinary tract urothelial carcinoma. Am J Kidney Dis 2007;50:743-753.

10. Buse S, Hach CE, Alexandrov A, et al. Simultaneous en-bloc robot-assisted radical cystectomy and nephro-ureterectomy: Technique description, outcomes, and literature summary. J Robotic Surg 2016;10:315-322.

11. Tseng SF, Chuang YC, Yang WC. Long-term outcome of radical cystectomy in ESDR patients with bladder urothelial carcinoma. Int Urol Nephrol 2011;43:1067-1071.

12. Barros R, Frota R, Stein RJ, et al. Simultaneous laparoscopic nephroureterectomy and cystectomy: A preliminary report. Int Braz J Urol 2008;34:413-421.

13. Pérez-Utrilla Pérez M, Aguilera Bazán A, Alonso Dorrego JM, et al. Simultaneous cystectomy and nephroureterectomy due to synchronous upper urinary tract tumors and invasive bladder cancer: Open and laparoscopic approaches. Curr Urol 2012;6:76-81.

14. Quek ML, Stein JP, Daneshmand S, et al. A critical analysis of perioperative mortality from radical cystectomy. J Urol 2006;175:886-890.

15. Novara G, Catto JWF, Wilson T, et al. Systematic review and cumulative analysis of perioperative outcomes and complications after robot-assisted radical cystectomy. Eur Urol 2015;67(3):376-401. 
16. Bream MJ, Maurice MJ, Altschuler J, et al. Increased use of cystectomy in patients 75 and older: A contemporary analysis of survival and perioperative outcomes from the national cancer database. Urology 2017;100:72-78.

17. Bochner BH, Dalbagni G, Marzouk KH, et al. Randomized trial comparing open radical cystectomy and robot-assisted laparoscopic radical cystectomy: Oncologic outcomes. Eur Urol 2018;74:465-471.

18. Gschwend JE, Heck MM, Lehmann J, et al. Extended versus limited lymph node dissection in bladder cancer patients undergoing radical cystectomy: Survival results from a prospective, randomized trial. Eur Urol 2019;75:604-611.

19. Stimson CJ, Chang SS, Barocas DA, et al. Early and late perioperative outcomes following radical cystectomy: 90-day readmissions, morbidity and mortality in a contemporary series. J Urol 2010;184:1296-1300.

20. Raman JD, Jafri SM. Complications following radical nephroureterectomy. Curr Urol Rep 2016[cited 2021 Sept 13];17(5):[about 5p.]. https://doiorg.foyer.swmed.edu/10.1007/s11934-016-0595-1. Accessed September 13, 2021.

21. Margulis V, Shariat SF, Matin SF, et al. Outcomes of radical nephroureterectomy: A series from the upper tract urothelial carcinoma collaboration. Cancer. 2009;115:12241233.

22. Gakis G, Black PC, Bochner BH, et al. Systematic review on the fate of the remnant urothelium after radical cystectomy. Eur Urol 2017;71:545-557.

23. Fahmy O, Khairul-Asri MG, Schubert T, et al. Urethral recurrence after radical cystectomy for urothelial carcinoma: A systematic review and meta-analysis. Urol Oncol 2018;36:54-59.

24. Khanna A, Zganjar A, Lyon T, et al. A contemporary analysis of urethral recurrence following radical cystectomy. J Urol 2021;206:970-977.

25. Boorjian SA, Kim SP, Weight CJ, et al. Risk factors and outcomes of urethral recurrence following radical cystectomy. Eur Urol 2011;60:1266-1272.

26. Huguet J, Monllau V, Sabaté S, et al. Diagnosis, risk factors, and outcome of urethral recurrences following radical cystectomy for bladder cancer in 729 male patients. Eur Urol 2008;53:785-793. 


\section{Figures and Tables}

\begin{tabular}{|l|l|}
\hline \multicolumn{2}{|l|}{$\begin{array}{l}\text { Table 1. Clinical and pathological characteristic of 27 patients who } \\
\text { underwent combined radical cystectomy/radical nephroureterectomy }\end{array}$} \\
\hline Variable & n (\%) or median (IQR) \\
\hline Total & 27 \\
\hline Age, years & $71(66-75)$ \\
\hline Sex & \\
\hline Male & $22(81 \%)$ \\
\hline Female & $5(19 \%)$ \\
\hline Race & \\
\hline White & $24(89 \%)$ \\
\hline Black & $1(3.7 \%)$ \\
\hline Hispanic & $1(3.7 \%)$ \\
\hline Asian & $1(3.7 \%)$ \\
\hline Smoking status & \\
\hline Never smoker & $8(30 \%)$ \\
\hline Prior smoker & $17(63 \%)$ \\
\hline Current smoker & $2(7.4 \%)$ \\
\hline Lynch syndrome & $2(7.4 \%)$ \\
\hline BMI & \\
\hline Mean ( \pm SD) & $28( \pm 4.4)$ \\
\hline ASA score & \\
\hline 1 & $0(0 \%)$ \\
\hline 2 & $4(15 \%)$ \\
\hline 3 & $21(78 \%)$ \\
\hline 4 & $2(7.4 \%)$ \\
\hline Charlson comorbidity index & \\
\hline $0-2$ & $4(15 \%)$ \\
\hline $3-5$ & $11(41 \%)$ \\
\hline $6-8$ & $10(37 \%)$ \\
\hline$\geq 9$ & $2(7.4 \%)$ \\
\hline Indication for nephrouretectomy & \\
\hline Upper tract urothelial carcinoma & $13(48 \%)$ \\
\hline Non-functional renal unit & $14(52 \%)$ \\
\hline Indication for cystectomy & \\
\hline De novo high-risk NMIBC & $2(7.4 \%)$ \\
\hline & \\
\hline
\end{tabular}




\begin{tabular}{|l|l|}
\hline De novo MIBC & $11(41 \%)$ \\
\hline Treatment-refractory NMIBC & $13(48 \%)$ \\
\hline Progressive NIMBC & $1(3.7 \%)$ \\
\hline Prior history of NMIBC & $14(52 \%)$ \\
\hline Interval since first diagnosis (months) & $60(18-67)$ \\
\hline Bacille Calmette-Guérin treatment & $13(93 \%)$ \\
\hline Ta/Tis & $11(79 \%)$ \\
\hline T1 & $3(21 \%)$ \\
\hline Low-grade & $4(29 \%)$ \\
\hline High-grade & $10(71 \%)$ \\
\hline Received neoadjuvant chemotherapy & $8(30 \%)$ \\
\hline clinical tumor stage, bladder & \\
\hline cTa/Tis & $13(48 \%)$ \\
\hline cT1 & $4(15 \%)$ \\
\hline cT2+ & $10(37 \%)$ \\
\hline Clinical tumor stage, upper tract & \\
\hline cTa/Tis & $11(41 \%)$ \\
\hline cT1 & $1(3.7 \%)$ \\
\hline cT2+ & $1(3.7 \%)$ \\
\hline Nodal stage (pelvic and upper tract) & \\
\hline cN0 & $26(96 \%)$ \\
\hline cN+ & $1(3.7 \%)$ \\
\hline Metastatic stage & \\
\hline cM0 & $26(96 \%)$ \\
\hline cM1a & $0(0 \%)$ \\
\hline cM1b & $1(3.7 \%)$ \\
\hline ASA Amican Sociey of Ans & \\
\hline
\end{tabular}

ASA: American Society of Anesthesiology; IQR: interquartile range (25th-75th percentiles); MIBC: muscle-invasive bladder cancer; NMIBC: non-muscle-invasive bladder cancer; SD: standard deviation. 


\begin{tabular}{|l|l|}
\hline \multicolumn{2}{|l|}{ Table 2. Perioperative outcomes and tumor pathology } \\
\hline Variable & n (\%) or median (IQR) \\
\hline Laterality of nephroureterectomy & \\
\hline Left & $7(26 \%)$ \\
\hline Right & $15(55 \%)$ \\
\hline Bilateral & $5(19 \%)$ \\
\hline Patients functionally anephric postoperatively & $11(41 \%)$ \\
\hline Newly HD-dependent & $4(15 \%)$ \\
\hline EBL (cc) & $600(250-1025)$ \\
\hline Operative time (min) & \\
\hline Diversion performed & $405(379-441)$ \\
\hline No diversion performed & $346(247-376)$ \\
\hline LOS (days) & $6(4.5-9)$ \\
\hline Transfusion (total for hospital stay) (units pRBC) & $2(0-4)$ \\
\hline Pathological tumor stage, bladder & \\
\hline pTa/Tis & $7(26 \%)$ \\
\hline pT1 & $3(11 \%)$ \\
\hline pT2 & $4(15 \%)$ \\
\hline pT3 & $3(11 \%)$ \\
\hline pT4 & $6(22 \%)$ \\
\hline Pathological tumor stage, upper tract & \\
\hline pTa/Tis & $7(26 \%)$ \\
\hline pT1 & $3(11 \%)$ \\
\hline pT2 & $2(7.4 \%)$ \\
\hline pT3 & $4(15 \%)$ \\
\hline pT4 & $0(0 \%)$ \\
\hline Pathological nodal stage (pelvic and upper tract) & \\
\hline pN0 & $20(74 \%)$ \\
\hline pN+ & $7(26 \%)$ \\
\hline Positive surgical margin (any) & $3(11 \%)$ \\
\hline $90-d a y$ mortality & $2(7.4 \%)$ \\
\hline $90-$ day re-admission & $7(26 \%)$ \\
\hline $90-$ day postoperative complication & \\
\hline Clavien-Dindo 1-2 & $12(44 \%)$ \\
\hline Clavien-Dindo 3-4 & $8(30 \%)$ \\
\hline
\end{tabular}

HD: hemodialysis; EBL: estimated blood loss; IQR: interquartile range $\left(25^{\text {th }}-75^{\text {th }}\right.$ percentiles); LOS:length of stay; pRBC: packed red blood cells. 


\begin{tabular}{|l|l|l|l|l|}
\hline \multicolumn{5}{|l|}{ Table 3. Perioperative outcomes by surgical approach } \\
\hline Variable, $n(\%)$ or median (IQR) & Open/open & MIS/open & MIS/MIS & p \\
\hline Number of patients & $13(48 \%)$ & $5(19 \%)$ & $9(33 \%)$ & - \\
\hline $\begin{array}{l}\text { Transfusion (total for hospital stay) (units } \\
\text { pRBC) }\end{array}$ & $3(2-5)$ & $0(0-0)$ & $1(0-2)$ & 0.004 \\
\hline Clavien-Dindo $\geq 3$ & $4(31 \%)$ & 0 & $3(33 \%)$ & 0.71 \\
\hline 90-day re-admission & $4(31 \%)$ & 0 & $3(33 \%)$ & 0.71 \\
\hline LOS (days) & $8(6-9)$ & $7(7-7)$ & $4(4-6)$ & 0.021 \\
\hline
\end{tabular}

IQR: interquartile range $\left(25^{\text {th }}-75^{\text {th }}\right.$ percentiles $)$; LOS: length of stay; MIS: minimally invasive surgery; pRBC: packed red blood cells.

\begin{tabular}{|l|l|}
\hline \multicolumn{2}{|l|}{ Table 4. Long-term survival and oncological outcomes } \\
\hline Variable & All patients \\
\hline Median followup (IQR) & $36(11-52)$ \\
\hline Overall survival & \\
\hline Median (95\% CI) & $47(41-53)$ \\
\hline 2-yr overall survival & $76 \%$ \\
\hline 5-yr overall survival & $42 \%$ \\
\hline Cancer-specific survival & \\
\hline Median (95\% CI) & $73(35-111)$ \\
\hline 2-yr cancer-specific survival & $76 \%$ \\
\hline 5-yr cancer-specific survival & $51 \%$ \\
\hline Progression-free survival & \\
\hline Median (95\% CI) & $30(16-44)$ \\
\hline 2-yr progression-free survival & $57 \%$ \\
\hline 5-yr progression-free survival & $41 \%$ \\
\hline Urethral recurrence & \\
\hline Incidence (\%) & $6(27 \%)$ \\
\hline
\end{tabular}

CI: confidence interval; IQR: interquartile range. 